\title{
Scientific Research and Social Well-being
}

$I^{N}$ his University Research Lecture, 1935-36, delivered at the University of Colorado, O. C. Lester, discussing the development and present status of graduate study and research in the University of Colorado, emphasized the importance of the spirit which animated the Graduate School and the attitude of the faculty to original creative work. The University of Colorado Studies was started chiefly as a medium for the publication of original work by members of the faculty and has been the chief medium of exchange with other universities, and gives important encouragement to creative work by affording a medium for publication.

Another obligation of the university to society is to maintain the supply of trained investigators, and the University's research fellowships and scholarships are designed to assist in discharging this obligation. It is not easy to evaluate the contributions of the faculty to research or other forms of creative scholarship, but the increasing demand for advanced work has led to the appointment of a Council of Research, one of the recommendations of which has led to the inauguration of an annual research lecture, of which Mr. Lester's is the first.

In the remainder of his lecture, $\mathrm{Mr}$. Lester discussed the relation of research, the university and the social order, and particularly the question whether scientific research workers should not modify their traditional attitude of being concerned only with discovery and not with its results to society or with the social consequences of scientific progress in general. The purpose of research is the betterment of human life, and on the whole that has been its effect. Apart from the comforts and conveniences beyond the reach of the rich a hundred years ago which are now possible for the average person, research has led to the eradication or alleviation of many ills of the body. Better understanding of the many factors affecting the health and vigour of both body and mind has led to the development of preventive measures which are becoming more and more effective. Reviewing briefly the organization and support of research, he stressed the supreme importance of the university in fundamental research, both as affording the right opportunity and atmosphere and in maintaining an adequate supply of investigators. The problems of modern civilization are too numerous, too varied, too complex to be solved by a few men, however capable, and the number of research workers, their technical training and the resources at their command required increasing in proportion.

Mr. Lester does not agree that we should be better off if much of our modern science and the appliances growing out of it were scrapped. If scientific advances have disturbed and continue to disturb the economic status quo, the remedy lies rather in attaining comparable and co-ordinating progress in the general field of the social sciences. Failure of progress here is indeed largely due to the inability of those who have reached well-founded conclusions to get a hearing, or even to unreasoning opposition to change. We need research on how to use the results of research for the general good, and in such work universities are well qualified to take a leading part. Not merely the general good but also scientific and industrial progress depend on a better social balance. Patchwork emergency methods and methods of political expediency are wholly inadequate for solving complex social problems and must be abandoned for thoughtful planning based upon thorough investigation.

\section{Some French Industrial Laboratories}

$\mathrm{T}$ HE University of Algiers has a small, but wellequipped, industrial testing laboratory under the direction of Prof. L. Vérain, professor of physics in the University. In a lecture entitled "Les Laboratoires industriels", delivered by Prof. Vérain, it is recorded that in 1935, some eight hundred certificates were issued by the Laboratory, the fees received being 56,000 francs.

The Laboratory's interests are many sided: electrical standards, electrotechnics and photometry; a wind tunnel; hydraulics; heat engines and refrigeration plants; the testing of wood, metal, cements, concretes, plasters and road materials; thermal conductivity ; acoustic insulation; absolute pressure ; viscosity ; and X-rays. In connexion with the testing of accelerometers, it is remarked that in Germany a car which cannot decelerate at not less than four metres per sec. per sec. is the subject of penalties if used after warning.

In his lecture, Prof. Vérain remarks that industrial laboratories date from the latter part of last century, when scientific methods were introduced into industry, and he traces their utility in the various stages of development of a process, or in the manufacture of a material or other product. Large works, especially chemical, metallurgical or engineering, commonly have their own laboratories.

In France, the first industrial laboratory was that of the École Nationale des Ponts et Chaussées, the chemical laboratory of which was opened in 1831. The city of Paris set up an engineering laboratory in 1869, and its specifications are still accepted by other organizations. The Laboratoire d'Essais du Conservatoire National des Arts et Métiers, founded in 1900, has five departments, but is not concerned with electrical measurements, for which a laboratory was founded in 1888, under the direction of the late M. Janet. This latter includes a radio laboratory, an engineering testing laboratory, and a co-ordinating office. The French Standards Association (AFNor) has issued some 800-1,000 standards, which may be contrasted with the 5,000 prepared by the corresponding German association. 